FUNCTION SPACES XII

BANACH CENTER PUBLICATIONS, VOLUME 119

INSTITUTE OF MATHEMATICS

POLISH ACADEMY OF SCIENCES

WARSZAWA 2019

\title{
SOME QUANTITATIVE RESULT ON COMPACT EMBEDDINGS IN SMOOTHNESS MORREY SPACES ON BOUNDED DOMAINS; AN APPROACH VIA INTERPOLATION
}

\author{
DOROTHEE D. HAROSKE \\ Institute of Mathematics, Friedrich Schiller University Jena \\ 07r37 Jena, Germany \\ E-mail: dorothee.haroske@uni-jena.de \\ LESZEK SKRZYPCZAK \\ Faculty of Mathematics $\&$ Computer Science, Adam Mickiewicz University \\ ul. Uniwersytetu Poznańskiego 4, 61-614 Poznań, Poland \\ E-mail: lskrzyp@amu.edu.pl
}

\begin{abstract}
We study the compact embedding between smoothness Morrey spaces on bounded domains and characterise its entropy numbers and approximation numbers in some cases.
\end{abstract}

1. Introduction. Let $\Omega \subset \mathbb{R}^{d}$ be a bounded $C^{\infty}$ domain. The main purpose of this short paper is to study the compactness of the embeddings

$$
\operatorname{id}_{\mathcal{N}}: \mathcal{N}_{u_{1}, p_{1}, q_{1}}^{s_{1}}(\Omega) \rightarrow \mathcal{N}_{u_{2}, p_{2}, q_{2}}^{s_{2}}(\Omega) \quad \text { and } \quad \operatorname{id}_{\mathcal{E}}: \mathcal{E}_{u_{1}, p_{1}, q_{1}}^{s_{1}}(\Omega) \rightarrow \mathcal{E}_{u_{2}, p_{2}, q_{2}}^{s_{2}}(\Omega),
$$

where $\mathcal{N}_{u, p, q}^{s}(\Omega)$ and $\mathcal{E}_{u, p, q}^{s}(\Omega)$ are Morrey smoothness spaces, with $s_{i} \in \mathbb{R}, 0<p_{i} \leq$ $u_{i}<\infty, 0<q_{i} \leq \infty, i=1,2$. Such spaces have been studied intensely in the past couple of years opening a wide field of possible applications. The Besov-Morrey spaces $\mathcal{N}_{u, p, q}^{s}\left(\mathbb{R}^{d}\right)$ were introduced in [15] by Kozono and Yamazaki and used by them and Mazzucato [16] to study Navier-Stokes equations. Corresponding Triebel-Lizorkin-Morrey spaces $\mathcal{E}_{u, p, q}^{s}\left(\mathbb{R}^{d}\right)$ were introduced in [29] by Tang and $\mathrm{Xu}$, where the authors established

2010 Mathematics Subject Classification: Primary 46E35; Secondary 41A46, 46B06.

Key words and phrases: Smoothness Morrey spaces on bounded domains, compact embeddings, entropy numbers, approximation numbers.

The paper is in final form and no version of it will be published elsewhere. 
the Morrey version of Fefferman-Stein vector-valued inequality. Properties of these spaces such as wavelet characterisations were studied in the papers by Sawano [22, 23], Sawano and Tanaka 25, 26], and Rosenthal [21]. Furthermore, the series [10, 11, 12] deals with some limiting embedding properties.

Other important, closely related scales of spaces are the so-called Besov-type spaces $B_{p, q}^{s, \tau}\left(\mathbb{R}^{d}\right)$ and Triebel-Lizorkin-type spaces $F_{p, q}^{s, \tau}\left(\mathbb{R}^{d}\right)$, introduced in [37], as well as the local and hybrid spaces dealt with in 33 and 34 . Both subjects are meanwhile studied in great detail, with interesting applications. But this will be out of the scope of this short note. We refer to the above monographs as well as to the fine surveys by Sickel [27, 28, and the recent papers [35, 36].

In [10, 12] we characterised the continuity of the embeddings

$$
\text { id }: \mathcal{N}_{u_{1}, p_{1}, q_{1}}^{s_{1}}\left(\mathbb{R}^{d}\right) \rightarrow \mathcal{N}_{u_{2}, p_{2}, q_{2}}^{s_{2}}\left(\mathbb{R}^{d}\right) \quad \text { and } \quad \text { id }: \mathcal{E}_{u_{1}, p_{1}, q_{1}}^{s_{1}}\left(\mathbb{R}^{d}\right) \rightarrow \mathcal{E}_{u_{2}, p_{2}, q_{2}}^{s_{2}}\left(\mathbb{R}^{d}\right),
$$

where $s_{i} \in \mathbb{R}, 0<p_{i} \leq u_{i}<\infty, 0<q_{i} \leq \infty, i=1,2$. But these embeddings can never be compact. However, turning to spaces on bounded domains, we obtained in [11] and [12] necessary and sufficient conditions for the corresponding embeddings (1) to be compact: this is the case if and only if

$$
\frac{s_{1}-s_{2}}{d}>\max \left\{0, \frac{1}{u_{1}}-\frac{1}{u_{2}}, \frac{p_{1}}{u_{1}}\left(\frac{1}{p_{1}}-\frac{1}{p_{2}}\right)\right\} .
$$

Recall that in case of $p_{i}=u_{i}, i=1,2$, we return to the classical situation of Besov and Triebel-Lizorkin spaces, since $B_{p, q}^{s}=\mathcal{N}_{p, p, q}^{s}$ and $F_{p, q}^{s}=\mathcal{E}_{p, p, q}^{s}$. Then the above findings (2) are in perfect agreement with that well-known situation where

$$
\operatorname{id}_{B}: B_{p_{1}, q_{1}}^{s_{1}}(\Omega) \rightarrow B_{p_{2}, q_{2}}^{s_{2}}(\Omega) \text { compact } \Longleftrightarrow \frac{s_{1}-s_{2}}{d}>\max \left\{0, \frac{1}{p_{1}}-\frac{1}{p_{2}}\right\},
$$

similarly for $\operatorname{id}_{F}: F_{p_{1}, q_{1}}^{s_{1}}(\Omega) \rightarrow F_{p_{2}, q_{2}}^{s_{2}}(\Omega)$. However, a lot more about the compactness of these embeddings is known in the 'classical' situation. The 'degree of compactness' as reflected by the asymptotic behaviour of the corresponding entropy and approximation numbers, is well-known; for the definition of entropy and approximation numbers as well as further applications of these concepts we refer to Section 2.3 below. The result for entropy numbers obtained in [5, 6] reads as

$$
e_{k}\left(\operatorname{id}_{B}: B_{p_{1}, q_{1}}^{s_{1}}(\Omega) \rightarrow B_{p_{2}, q_{2}}^{s_{2}}(\Omega)\right) \sim k^{-\left(s_{1}-s_{2}\right) / d}, \quad k \in \mathbb{N},
$$

where we always assume (3) to hold. The corresponding results for $F$-spaces and in case of approximation numbers can be found in Section 2.3 below.

However, as far as we know, there are almost no results for entropy and approximation numbers of the compact embeddings (1). We contributed a little to this subject in [35], in the recent paper [1] one can find approximation number results for the periodic case with the target space $L_{\infty}$. Apart from this we are not aware of any such outcome.

It is the purpose of this short note to obtain some first result for the corresponding entropy numbers of the compact embeddings in (1), though we cannot cover all possible cases with our method here. We want to demonstrate that at least for sufficiently large smoothness differences the classical asymptotic behaviour of the entropy numbers in (4) 
will survive the Morreyfication of the underlying spaces. More precisely, we can prove in Theorem 3.1 that under the additional assumption $s_{1}-s_{2}>d \max \left\{0, \frac{1}{p_{1}}-\frac{1}{u_{2}}\right\}$,

$$
e_{k}\left(\operatorname{id}_{\mathcal{N}}: \mathcal{N}_{u_{1}, p_{1}, q_{1}}^{s_{1}}(\Omega) \rightarrow \mathcal{N}_{u_{2}, p_{2}, q_{2}}^{s_{2}}(\Omega)\right) \sim k^{-\left(s_{1}-s_{2}\right) / d}, \quad k \in \mathbb{N} .
$$

However, we do not claim that this classical behaviour will be true in all cases given by (2), but this discussion is out of the scope of the present note. But to prove the above result (and counterparts for $\mathcal{E}_{u, p, q}^{s}$ spaces and approximation numbers) we benefit from well-known methods like embeddings, interpolation and their interplay with entropy numbers. Their strength in this situation is demonstrated. Moreover, in case of entropy numbers this seem to be the first sharp results at all.

The paper is organised as follows. In Section 2 we briefly introduce the corresponding function spaces and the concepts of entropy and approximation numbers, while Section 3 contains our new results and their proofs.

2. Preliminaries. First we fix some notation. By $\mathbb{N}$ we denote the set of natural numbers, by $\mathbb{N}_{0}$ the set $\mathbb{N} \cup\{0\}$. For $a \in \mathbb{R}$, let $a_{+}:=\max \{a, 0\}$.

All unimportant positive constants will be denoted by $C$, occasionally with subscripts. By the notation $A \lesssim B$, we mean that there exists a positive constant $C$ such that $A \leq C B$, whereas the symbol $A \sim B$ stands for $A \lesssim B \lesssim A$.

Given two (quasi-)Banach spaces $X$ and $Y$, we write $X \hookrightarrow Y$ if $X \subset Y$ and the natural embedding of $X$ into $Y$ is continuous.

2.1. Smoothness Morrey spaces on $\mathbb{R}^{d}$. Let $\mathcal{S}\left(\mathbb{R}^{d}\right)$ be the set of all Schwartz functions on $\mathbb{R}^{d}$, endowed with the usual topology, and denote by $\mathcal{S}^{\prime}\left(\mathbb{R}^{d}\right)$ its topological dual, namely, the space of all bounded linear functionals on $\mathcal{S}\left(\mathbb{R}^{d}\right)$ endowed with the weak *-topology. For all $f \in \mathcal{S}\left(\mathbb{R}^{d}\right)$ or $\mathcal{S}^{\prime}\left(\mathbb{R}^{d}\right)$, we use $\widehat{f}$ to denote its Fourier transform, and $f^{\vee}$ for its inverse.

Let $\varphi_{0}, \varphi \in \mathcal{S}\left(\mathbb{R}^{d}\right)$ be such that

$$
\operatorname{supp} \widehat{\varphi_{0}} \subset\left\{\xi \in \mathbb{R}^{d}:|\xi| \leq 2\right\}, \quad\left|\widehat{\varphi_{0}}(\xi)\right| \geq C \text { if }|\xi| \leq 5 / 3
$$

and

$$
\operatorname{supp} \widehat{\varphi} \subset\left\{\xi \in \mathbb{R}^{d}: 1 / 2 \leq|\xi| \leq 2\right\} \quad \text { and } \quad|\widehat{\varphi}(\xi)| \geq C \text { if } 3 / 5 \leq|\xi| \leq 5 / 3,
$$

where $C$ is a positive constant. In what follows, for all $\varphi \in \mathcal{S}\left(\mathbb{R}^{d}\right)$ and $j \in \mathbb{N}, \varphi_{j}(\cdot):=$ $2^{j d} \varphi\left(2^{j} \cdot\right)$.

We introduce smoothness spaces of Morrey type. Recall first that the Morrey space $\mathcal{M}_{u, p}\left(\mathbb{R}^{d}\right), 0<p \leq u<\infty$, is defined to be the set of all locally $p$-integrable functions $f \in L_{p}^{\text {loc }}\left(\mathbb{R}^{d}\right)$ such that

$$
\left\|f \mid \mathcal{M}_{u, p}\left(\mathbb{R}^{d}\right)\right\|:=\sup _{x \in \mathbb{R}^{d}, R>0} R^{d / u-d / p}\left[\int_{B(x, R)}|f(y)|^{p} \mathrm{~d} y\right]^{1 / p}<\infty .
$$

REMARK 2.1. The spaces $\mathcal{M}_{u, p}\left(\mathbb{R}^{d}\right)$ are quasi-Banach spaces (Banach spaces for $p \geq 1$ ). They originated from Morrey's study on PDE (see [17]) and are part of the wider class of Morrey-Campanato spaces; cf. [18]. They can be considered as a complement to $L_{p}$ spaces, since $\mathcal{M}_{p, p}\left(\mathbb{R}^{d}\right)=L_{p}\left(\mathbb{R}^{d}\right)$ with $p \in(0, \infty)$, extended by $\mathcal{M}_{\infty, \infty}\left(\mathbb{R}^{d}\right)=L_{\infty}\left(\mathbb{R}^{d}\right)$. 
In a parallel way one can define the spaces $\mathcal{M}_{\infty, p}\left(\mathbb{R}^{d}\right), p \in(0, \infty)$, but using the Lebesgue differentiation theorem, one arrives at $\mathcal{M}_{\infty, p}\left(\mathbb{R}^{d}\right)=L_{\infty}\left(\mathbb{R}^{d}\right)$. Moreover, $\mathcal{M}_{u, p}\left(\mathbb{R}^{d}\right)=\{0\}$ for $u<p$, and for $0<p_{2} \leq p_{1} \leq u<\infty$,

$$
L_{u}\left(\mathbb{R}^{d}\right)=\mathcal{M}_{u, u}\left(\mathbb{R}^{d}\right) \hookrightarrow \mathcal{M}_{u, p_{1}}\left(\mathbb{R}^{d}\right) \hookrightarrow \mathcal{M}_{u, p_{2}}\left(\mathbb{R}^{d}\right) .
$$

Now we present the smoothness spaces of Morrey type in which we are interested.

Definition 2.2. Let $0<p \leq u<\infty$ or $p=u=\infty$. Let $q \in(0, \infty], s \in \mathbb{R}$ and $\varphi_{0}$, $\varphi \in \mathcal{S}\left(\mathbb{R}^{d}\right)$ be as in (6) and $(7)$, respectively.

(i) The Besov-Morrey space $\mathcal{N}_{u, p, q}^{s}\left(\mathbb{R}^{d}\right)$ is defined to be the set of all distributions $f \in \mathcal{S}^{\prime}\left(\mathbb{R}^{d}\right)$ such that

$$
\left\|f \mid \mathcal{N}_{u, p, q}^{s}\left(\mathbb{R}^{d}\right)\right\|:=\left[\sum_{j=0}^{\infty} 2^{j s q}\left\|\varphi_{j} * f \mid \mathcal{M}_{u, p}\left(\mathbb{R}^{d}\right)\right\|^{q}\right]^{1 / q}<\infty
$$

with the usual modification made in case of $q=\infty$.

(ii) Let $u \in(0, \infty)$. The Triebel-Lizorkin-Morrey space $\mathcal{E}_{u, p, q}^{s}\left(\mathbb{R}^{d}\right)$ is defined to be the set of all distributions $f \in \mathcal{S}^{\prime}\left(\mathbb{R}^{d}\right)$ such that

$$
\left\|f\left|\mathcal{E}_{u, p, q}^{s}\left(\mathbb{R}^{d}\right)\|:=\|\left[\sum_{j=0}^{\infty} 2^{j s q}\left|\varphi_{j} * f\right|^{q}\right]^{1 / q}\right| \mathcal{M}_{u, p}\left(\mathbb{R}^{d}\right)\right\|<\infty
$$

with the usual modification made in case of $q=\infty$.

REMARK 2.3. In addition to our historical remarks in the introduction, we would like to refer to the most systematic and general approach to spaces of this type: it can be found in the book [37] or in the survey papers by Sickel [27, 28]. We recommend the monograph and the surveys for further references on this subject. Mazzucato has shown in [16, Prop. 4.1] that

$$
\mathcal{E}_{u, p, 2}^{0}\left(\mathbb{R}^{d}\right)=\mathcal{M}_{u, p}\left(\mathbb{R}^{d}\right), \quad 1<p \leq u<\infty .
$$

Convention. We adopt the nowadays usual custom to write $A_{p, q}^{s}$ instead of $B_{p, q}^{s}$ or $F_{p, q}^{s}$, and $\mathcal{A}_{u, p, q}^{s}$ instead of $\mathcal{N}_{u, p, q}^{s}$ or $\mathcal{E}_{u, p, q}^{s}$, respectively, when both scales of spaces are meant simultaneously in some context.

REMARK 2.4. The spaces $\mathcal{A}_{u, p, q}^{s}\left(\mathbb{R}^{d}\right)$ are independent of the particular choices of $\varphi_{0}, \varphi$ appearing in their definitions. They are quasi-Banach spaces (Banach spaces for $p, q \geq 1$ ), and $\mathcal{S}\left(\mathbb{R}^{d}\right) \hookrightarrow \mathcal{A}_{u, p, q}^{s}\left(\mathbb{R}^{d}\right) \hookrightarrow \mathcal{S}^{\prime}\left(\mathbb{R}^{d}\right)$. Moreover, for $u=p$ we re-obtain the usual Besov and Triebel-Lizorkin spaces, $\mathcal{A}_{p, p, q}^{s}\left(\mathbb{R}^{d}\right)=A_{p, q}^{s}\left(\mathbb{R}^{d}\right)$. There exists extensive literature on such spaces; we refer, in particular, to the series of monographs [30, 31, 32] for a comprehensive treatment. In case of $u<p$ we have $\mathcal{A}_{u, p, q}^{s}\left(\mathbb{R}^{d}\right)=\{0\}$. We occasionally benefit from the elementary embeddings

$$
B_{p, \min \{p, q\}}^{s}\left(\mathbb{R}^{d}\right) \hookrightarrow F_{p, q}^{s}\left(\mathbb{R}^{d}\right) \hookrightarrow B_{p, \max \{p, q\}}^{s}\left(\mathbb{R}^{d}\right),
$$

where $p \in(0, \infty), q \in(0, \infty]$ and $s \in \mathbb{R}$. The result for spaces $\mathcal{A}_{u, p, q}^{s}$ is different: Sawano proved in 22$]$ that, for $s \in \mathbb{R}$ and $0<p<u<\infty$,

$$
\mathcal{N}_{u, p, \min \{p, q\}}^{s}\left(\mathbb{R}^{d}\right) \hookrightarrow \mathcal{E}_{u, p, q}^{s}\left(\mathbb{R}^{d}\right) \hookrightarrow \mathcal{N}_{u, p, \infty}^{s}\left(\mathbb{R}^{d}\right),
$$

where, for the latter embedding, $r=\infty$ cannot be improved. 
2.2. Function spaces on domains. Unless otherwise stated, we shall assume that $\Omega$ is a bounded $C^{\infty}$ domain in $\mathbb{R}^{d}$. We consider smoothness Morrey spaces on $\Omega$ defined by restriction. Let $\mathcal{D}(\Omega)$ be the set of all infinitely differentiable functions supported in $\Omega$ and denote by $\mathcal{D}^{\prime}(\Omega)$ its dual. Since we are able to define the extension operator ext : $\mathcal{D}(\Omega) \rightarrow \mathcal{S}\left(\mathbb{R}^{d}\right)$, the restriction operator re : $\mathcal{S}^{\prime}\left(\mathbb{R}^{d}\right) \rightarrow \mathcal{D}^{\prime}(\Omega)$ can be defined naturally as an adjoint operator

$$
\langle\operatorname{re}(f), \varphi\rangle=\langle f, \operatorname{ext}(\varphi)\rangle, \quad f \in \mathcal{S}^{\prime}\left(\mathbb{R}^{d}\right),
$$

where $\varphi \in \mathcal{D}(\Omega)$. We will write $\left.f\right|_{\Omega}=\operatorname{re}(f)$.

Definition 2.5. Let $0<p \leq u<\infty$ or $p=u=\infty, q \in(0, \infty]$ and $s \in \mathbb{R}$ (with $u<\infty$ in the case of $\mathcal{A}=\mathcal{E})$. Then $\mathcal{A}_{u, p, q}^{s}(\Omega)$ is defined by

$$
\mathcal{A}_{u, p, q}^{s}(\Omega):=\left\{f \in \mathcal{D}^{\prime}(\Omega): f=\left.g\right|_{\Omega} \text { for some } g \in \mathcal{A}_{u, p, q}^{s}\left(\mathbb{R}^{d}\right)\right\}
$$

endowed with the quasi-norm

$$
\left\|f \mid \mathcal{A}_{u, p, q}^{s}(\Omega)\right\|:=\inf \left\{\left\|g\left|\mathcal{A}_{u, p, q}^{s}\left(\mathbb{R}^{d}\right) \|: f=g\right|_{\Omega}, g \in \mathcal{A}_{u, p, q}^{s}\left(\mathbb{R}^{d}\right)\right\} .\right.
$$

REMARK 2.6. The spaces $\mathcal{A}_{u, p, q}^{s}(\Omega)$ are quasi-Banach spaces (Banach spaces for $p, q \geq 1$ ). When $u=p$ we re-obtain the usual Besov and Triebel-Lizorkin spaces defined on bounded smooth domains. Several properties of the spaces $\mathcal{A}_{u, p, q}^{s}(\Omega)$, including the extension property, were studied in [24]. Embeddings within spaces in this latter scale as well as to classical spaces like $C(\Omega)$ or $L_{r}(\Omega)$ were investigated in [11, 12. In [9] we studied the question under what assumptions these spaces consist of regular distributions only.

In case of the target space $L_{\infty}(\Omega)$ we obtained in [11, 12] the following result.

Proposition 2.7. Let $s \in \mathbb{R}, 0<p<u<\infty$ and $q \in(0, \infty]$. Then

$$
\mathcal{A}_{u, p, q}^{s}(\Omega) \hookrightarrow L_{\infty}(\Omega) \quad \text { is compact if and only if } s>\frac{d}{u} .
$$

The next theorem regards real interpolation of Besov-Morrey spaces on $\Omega$. It can be proved similarly to the proof of [32, Thm. 1.110], relying on the corresponding assertions with $\Omega$ replaced by $\mathbb{R}^{d}$ that can be found in [28, Thm. 2.2, Prop. 2.3], and on the existence of a common extension operator, cf. [24, Thm. 5.4].

TheOREM 2.8. Let $u, q, q_{0}, q_{1} \in(0, \infty]$ and $\theta \in(0,1)$.

(i) Let $0<p \leq u<\infty$ and $s_{0}, s_{1} \in \mathbb{R}$ with $s_{0} \neq s_{1}$. Then

$$
\mathcal{N}_{u, p, q}^{s}(\Omega)=\left(\mathcal{N}_{u, p, q_{0}}^{s_{0}}(\Omega), \mathcal{N}_{u, p, q_{1}}^{s_{1}}(\Omega)\right)_{\theta, q} \quad \text { if } \quad s=(1-\theta) s_{0}+\theta s_{1} .
$$

(ii) Let $1 \leq p \leq u<\infty$ and $s \in \mathbb{R}$. Then

$$
\mathcal{N}_{u, p, q}^{s}(\Omega)=\left(\mathcal{N}_{u, p, q_{0}}^{s}(\Omega), \mathcal{N}_{u, p, q_{1}}^{s}(\Omega)\right)_{\theta, q} \quad \text { if } \quad \frac{1}{q}=\frac{1-\theta}{q_{0}}+\frac{\theta}{q_{1}} .
$$

Let us finally state our compactness result as obtained in [11] (for $\mathcal{A}=\mathcal{N}$ ) and [12] $($ for $\mathcal{A}=\mathcal{E})$. 
Theorem 2.9. Let $s_{1}, s_{2} \in \mathbb{R}, 0<q_{1}, q_{2} \leq \infty, 0<p_{1} \leq u_{1}<\infty, 0<p_{2} \leq u_{2}<\infty$. Then the embedding

$$
\operatorname{id}_{\mathcal{A}}: \mathcal{A}_{u_{1}, p_{1}, q_{1}}^{s_{1}}(\Omega) \hookrightarrow \mathcal{A}_{u_{2}, p_{2}, q_{2}}^{s_{2}}(\Omega)
$$

is compact if and only if

$$
\frac{s_{1}-s_{2}}{d}>\max \left\{0, \frac{1}{u_{1}}-\frac{1}{u_{2}}, \frac{p_{1}}{u_{1}}\left(\frac{1}{p_{1}}-\frac{1}{p_{2}}\right)\right\} .
$$

2.3. Entropy and approximation numbers. Let $A_{1}$ and $A_{2}$ be two complex (quasi-) Banach spaces and let $T$ be a linear and continuous operator from $A_{1}$ into $A_{2}$. If $T$ is compact, then for any given $\varepsilon>0$ there are finitely many balls in $A_{2}$ of radius $\varepsilon$ which cover the image $T U_{1}$ of the unit ball $U_{1}=\left\{a \in A_{1}:\left\|a \mid A_{1}\right\| \leq 1\right\}$.

Definition 2.10. Let $A_{1}$ and $A_{2}$ be two complex (quasi-) Banach spaces, $k \in \mathbb{N}$ and let $T: A_{1} \rightarrow A_{2}$ be a linear and continuous operator from $A_{1}$ into $A_{2}$.

(i) The $k$ th entropy number $e_{k}$ of $T$ is the infimum of all numbers $\varepsilon>0$ such that there exist $2^{k-1}$ balls in $A_{2}$ of radius $\varepsilon$ which cover $T U_{1}$.

(ii) The $k$ th approximation number $a_{k}$ of $T$ is the infimum of all numbers $\|T-S\|$ where $S$ runs through the collection of all continuous linear maps from $A_{1}$ to $A_{2}$ with $\operatorname{rank} S<k$,

$$
a_{k}(T)=\inf \left\{\|T-S\|: S \in \mathcal{L}\left(A_{1}, A_{2}\right), \operatorname{rank} S<k\right\} .
$$

For details and properties of approximation numbers we refer to [3, 4, 14, 20] (restricted to the case of Banach spaces), and [7] for some extensions to quasi-Banach spaces. Among other features we only want to mention the multiplicativity of entropy numbers and approximation numbers: let $A_{1}, A_{2}$ and $A_{3}$ be complex (quasi-) Banach spaces and $T_{1}: A_{1} \rightarrow A_{2}, T_{2}: A_{2} \rightarrow A_{3}$ two operators in the sense of Definition 2.10. Then

$$
\begin{aligned}
e_{k_{1}+k_{2}-1}\left(T_{2} \circ T_{1}\right) \leq e_{k_{1}}\left(T_{1}\right) e_{k_{2}}\left(T_{2}\right), \quad k_{1}, k_{2} \in \mathbb{N}, \\
\text { and } \quad a_{k_{1}+k_{2}-1}\left(T_{2} \circ T_{1}\right) \leq a_{k_{1}}\left(T_{1}\right) a_{k_{2}}\left(T_{2}\right), \quad k_{1}, k_{2} \in \mathbb{N} .
\end{aligned}
$$

Note that in general $\lim _{k \rightarrow \infty} e_{k}(T)=0$ if and only if $T$ is compact. The last equivalence justifies the saying that entropy numbers measure "how compact" an operator acts. Dealing with approximation numbers there is no complete counterpart in general; in particular, one only observes that $\lim _{n \rightarrow \infty} a_{n}(T)=0$ implies that $T$ is compact. It is known that it may happen that $\lim _{n \rightarrow \infty} a_{n}(T)=\alpha(T)>0$ for some compact $T \in \mathcal{L}(A, B)$ when $B$ fails to have the approximation property, see [4] and [19, Prop. 10.1.3, 10.1.4]. Many spaces possess that property, but there exist spaces without it, cf. [8], [19, Thm. 10.4.7].

Moreover, approximation numbers - unlike entropy numbers - can be regarded as special s-numbers, a concept introduced by Pietsch [19, Sect. 11].

Finally we recall the following property of entropy numbers proved in [13, Theorem 3.2] and [7, Theorem 1.3.2]. Let $A$ be a quasi-Banach space and let $\left\{B_{0}, B_{1}\right\}$ be an interpolation couple of quasi-Banach spaces. Let $0<\theta<1$ and let $B_{\theta}$ be a quasi-Banach space such that

$$
B_{0} \cap B_{1} \hookrightarrow B_{\theta} \hookrightarrow B_{0}+B_{1} \quad \text { (naturally quasi-normed) }
$$

and

$$
\left\|b\left|B_{\theta}\|\leq\| b\right| B_{0}\right\|^{1-\theta}\left\|b \mid B_{1}\right\|^{\theta} \quad \text { for all } \quad b \in B_{0} \cap B_{1} .
$$


Let $T \in \mathcal{L}\left(A, B_{0} \cap B_{1}\right)$. Then there is a number $c>0$ such that for all $k \in \mathbb{N}$,

$$
e_{2 k}\left(T: A \hookrightarrow B_{\theta}\right) \leq c e_{k}^{1-\theta}\left(T: A \hookrightarrow B_{0}\right) e_{k}^{\theta}\left(T: A \hookrightarrow B_{1}\right) .
$$

REMARK 2.11. We recall what is well-known in case of the embedding

$$
\operatorname{id}_{A}: A_{p_{1}, q_{1}}^{s_{1}}(\Omega) \rightarrow A_{p_{2}, q_{2}}^{s_{2}}(\Omega),
$$

where $-\infty<s_{2} \leq s_{1}<\infty, 0<p_{1}, p_{2} \leq \infty\left(p_{1}, p_{2}<\infty\right.$ in the $F$-case $), 0<q_{1}, q_{2} \leq \infty$, and the spaces $A_{p, q}^{s}(\Omega)$ are defined by restriction. Let

$$
\delta=s_{1}-s_{2}-d\left(\frac{1}{p_{1}}-\frac{1}{p_{2}}\right), \quad \delta_{+}=s_{1}-s_{2}-d\left(\frac{1}{p_{1}}-\frac{1}{p_{2}}\right)_{+} .
$$

Then $\operatorname{id}_{A}$ is compact when $\delta_{+}>0$; cf. [7, (2.5.1/10)]. The extension to values $p_{2}<p_{1}-$ compared with the $\mathbb{R}^{d}$-setting - is due to Hölder's inequality. In this situation Edmunds and Triebel proved in [5, 6] (see also [7, Thm. 3.3.3/2]) that

$$
e_{k}\left(\operatorname{id}_{A}\right) \sim k^{-\left(s_{1}-s_{2}\right) / d}, \quad k \in \mathbb{N},
$$

where $s_{1} \geq s_{2}, 0<p_{1}, p_{2} \leq \infty\left(p_{1}, p_{2}<\infty\right.$ in the $F$-case $), 0<q_{1}, q_{2} \leq \infty$, and $\delta_{+}>0$. In the case of approximation numbers the situation is more complicated; the result of Edmunds and Triebel in [7] Thm. 3.3.4], partly improved by Caetano [2], reads as

$$
a_{k}\left(\operatorname{id}_{A}\right) \sim k^{-\delta_{+} / d-\varkappa}, \quad k \in \mathbb{N},
$$

with

$$
\varkappa=\left(\frac{\min \left(p_{1}^{\prime}, p_{2}\right)}{2}-1\right)_{+} \cdot \min \left(\frac{\delta}{d}, \frac{1}{\min \left(p_{1}^{\prime}, p_{2}\right)}\right),
$$

and $\delta$ is given by (16). The above asymptotic result is almost complete now, apart from the restrictions that $\left(p_{1}, p_{2}\right) \neq(1, \infty)$ or $\frac{\delta}{d} \neq \frac{1}{\min \left(p_{1}^{\prime}, p_{2}\right)}$ when $0<p_{1}<2<p_{2} \leq \infty$. Note that $\varkappa=0$ unless $p_{1}<2<p_{2}$, and $\delta \geq \delta_{+}$with $\delta=\delta_{+}$if $p_{1} \leq p_{2}$.

3. The main results. We first want to estimate the entropy numbers of the compact embedding (11) in case of sufficiently 'large' difference of smoothness, and give some application to approximation numbers afterwards.

\subsection{Entropy numbers}

TheOREM 3.1. Let $s_{1}, s_{2} \in \mathbb{R}, 0<q_{1}, q_{2} \leq \infty, 0<p_{1} \leq u_{1}<\infty, 0<p_{2} \leq u_{2}<\infty$, with

$$
\frac{s_{1}-s_{2}}{d}>\max \left\{0, \frac{1}{u_{1}}-\frac{1}{u_{2}}, \frac{p_{1}}{u_{1}}\left(\frac{1}{p_{1}}-\frac{1}{p_{2}}\right)\right\} .
$$

(i) Assume, in addition, that

$$
\frac{s_{1}-s_{2}}{d}>\max \left\{0, \frac{1}{p_{1}}-\frac{1}{u_{2}}\right\} .
$$

Then

$$
e_{k}\left(\operatorname{id}_{\mathcal{A}}: \mathcal{A}_{u_{1}, p_{1}, q_{1}}^{s_{1}}(\Omega) \hookrightarrow \mathcal{A}_{u_{2}, p_{2}, q_{2}}^{s_{2}}(\Omega)\right) \sim k^{-\left(s_{1}-s_{2}\right) / d}, \quad k \in \mathbb{N} .
$$

(ii) If $u_{1} \geq u_{2}, p_{1} \geq p_{2}$ and $s_{1}-s_{2}>0$, then (22) is always true. 
Proof. Step 1. In view of the elementary embeddings (10, the independence of 21) and 22 from the fine parameters $q_{1}, q_{2}$, and the multiplicativity of entropy numbers [12], it is sufficient to prove the above result for spaces $\mathcal{A}_{u, p, q}^{s}=\mathcal{N}_{u, p, q}^{s}$.

Step 2. Next we benefit from the classical result (17) and use the following chain of embeddings,

$$
B_{u_{1}, q_{1}}^{s_{1}}(\Omega) \hookrightarrow \mathcal{N}_{u_{1}, p_{1}, q_{1}}^{s_{1}}(\Omega) \hookrightarrow B_{p_{1}, q_{1}}^{s_{1}}(\Omega) \hookrightarrow B_{u_{2}, q_{2}}^{s_{2}}(\Omega) \hookrightarrow \mathcal{N}_{u_{2}, p_{2}, q_{2}}^{s_{2}}(\Omega) \hookrightarrow B_{p_{2}, q_{2}}^{s_{2}}(\Omega) .
$$

We consider the embeddings

$$
\begin{aligned}
\operatorname{id}_{B}^{\text {out }}: B_{u_{1}, q_{1}}^{s_{1}}(\Omega) \hookrightarrow B_{p_{2}, q_{2}}^{s_{2}}(\Omega), \\
\operatorname{id}_{B}^{\text {int }}: B_{p_{1}, q_{1}}^{s_{1}}(\Omega) \hookrightarrow B_{u_{2}, q_{2}}^{s_{2}}(\Omega),
\end{aligned}
$$

and conclude from Remark 2.11 that id ${ }_{B}^{\text {out }}$ is compact if and only if $s_{1}-s_{2}>d\left(\frac{1}{u_{1}}-\frac{1}{p_{2}}\right)_{+}$ with

$$
e_{k}\left(\operatorname{id}_{B}^{\text {out }}\right) \sim k^{-\left(s_{1}-s_{2}\right) / d}, \quad k \in \mathbb{N},
$$

and $\mathrm{id}_{B}^{\mathrm{int}}$ is compact if and only if $s_{1}-s_{2}>d\left(\frac{1}{p_{1}}-\frac{1}{u_{2}}\right)+$ with

$$
e_{k}\left(\operatorname{id}_{B}^{\mathrm{int}}\right) \sim k^{-\left(s_{1}-s_{2}\right) / d}, \quad k \in \mathbb{N} .
$$

In particular, the compactness of $\mathrm{id}_{B}^{\text {int }}$ implies the compactness of $\mathrm{id}_{\mathcal{N}}$ which implies the compactness of $\mathrm{id}_{B}^{\text {out }}$. The multiplicativity of entropy numbers 12 yields

$$
e_{k}\left(\operatorname{id}_{B}^{\text {out }}\right) \leq c e_{k}\left(\operatorname{id}_{\mathcal{N}}\right) \leq c^{\prime} e_{k}\left(\operatorname{id}_{B}^{\text {int }}\right) .
$$

Hence, if $s_{1}-s_{2}>d\left(\frac{1}{p_{1}}-\frac{1}{u_{2}}\right)_{+} \geq d\left(\frac{1}{u_{1}}-\frac{1}{p_{2}}\right)_{+}$, then

$$
e_{k}\left(\operatorname{id}_{\mathcal{N}}\right) \sim k^{-\left(s_{1}-s_{2}\right) / d} .
$$

This completes the proof of $(\mathrm{i})$. Note that the lower estimate

$$
e_{k}\left(\operatorname{id}_{\mathcal{N}}\right) \geq c k^{-\left(s_{1}-s_{2}\right) / d}
$$

is always true in the compact case of Theorem 2.9 due to 28 and 26 .

Step 3. It remains to show (ii). In view of (30) only the upper estimate has to be considered. So far open is the case when

$$
\max \left\{0, \frac{1}{u_{1}}-\frac{1}{u_{2}}, \frac{p_{1}}{u_{1}}\left(\frac{1}{p_{1}}-\frac{1}{p_{2}}\right)\right\}<\frac{s_{1}-s_{2}}{d} \leq\left(\frac{1}{p_{1}}-\frac{1}{u_{2}}\right)_{+},
$$

where we have a compact embedding according to Theorem 2.9. It can only appear when $u_{1}<u_{2}, p_{1}<p_{2}$. Using interpolation arguments, that is, Theorem 2.8 together with the interpolation property of entropy numbers (15), we may extend 29 to all cases of 20 where

$$
u_{1} \geq u_{2}, \quad p_{1} \geq p_{2}, \quad s_{1}>s_{2} .
$$

Assume $u_{1} \geq u_{2}, p_{1} \geq p_{2}$ and $d\left(\frac{1}{p_{1}}-\frac{1}{u_{2}}\right)_{+} \geq s_{1}-s_{2}>0$, hence id $\mathcal{N}_{\mathcal{N}}$ is compact, but the situation is not covered by (i). Note that

$$
\operatorname{id}_{\mathcal{N}}^{1}: \mathcal{N}_{u_{1}, p_{1}, q_{1}}^{s_{1}}(\Omega) \rightarrow \mathcal{N}_{u_{2}, p_{2}, v_{1}}^{s_{1}}(\Omega)
$$

is continuous for $v_{1} \geq q_{1}$, hence $e_{k}\left(\mathrm{id}_{\mathcal{N}}^{1}\right) \leq c, k \in \mathbb{N}$. Now choose $\sigma$ such that

$$
\sigma<s_{1}-d\left(\frac{1}{p_{1}}-\frac{1}{u_{2}}\right) \leq s_{2}, \quad \text { hence } \quad s_{1}-\sigma>d\left(\frac{1}{p_{1}}-\frac{1}{u_{2}}\right)_{+} .
$$


Then (i) implies that

$$
e_{k}\left(\operatorname{id}_{\mathcal{N}}^{2}: \mathcal{N}_{u_{1}, p_{1}, q_{1}}^{s_{1}}(\Omega) \rightarrow \mathcal{N}_{u_{2}, p_{2}, v_{2}}^{\sigma}(\Omega)\right) \leq c k^{-\left(s_{1}-\sigma\right) / d} .
$$

On the other hand, Theorem 2.8 yields

$$
\mathcal{N}_{u_{2}, p_{2}, q_{2}}^{s_{2}}(\Omega)=\left(\mathcal{N}_{u_{2}, p_{2}, v_{1}}^{s_{1}}(\Omega), \mathcal{N}_{u_{2}, p_{2}, v_{2}}^{\sigma}(\Omega)\right)_{\theta, q_{2}} \quad \text { if } \quad s_{2}=(1-\theta) s_{1}+\theta \sigma,
$$

that is, $\theta\left(s_{1}-\sigma\right)=s_{1}-s_{2}$. But now the interpolation property 15 leads to

$$
e_{k}\left(\operatorname{id}_{\mathcal{N}}\right) \leq c e_{k}\left(\operatorname{id}_{\mathcal{N}}^{1}\right)^{1-\theta} e_{k}\left(\operatorname{id}_{\mathcal{N}}^{2}\right)^{\theta} \leq c^{\prime} k^{-\left(s_{1}-\sigma\right) / d \theta}=c^{\prime} k^{-\left(s_{1}-s_{2}\right) / d} .
$$

This completes the proof.

REMARK 3.2. Note that apart from case (ii) there always remains a gap between the situation covered by (i) and the precise condition (20), recall (31). Whenever $p_{1}<p_{2}$, or $p_{1} \geq p_{2}$ with $u_{1}<u_{2}$, the right-hand side in (31) is in general larger than the left-hand side. This means that the only extension of (i) we can cover by our method is described in (ii).

Now we can strengthen Proposition 2.7 for sufficiently large $s$ as follows.

Corollary 3.3. Let $s \in \mathbb{R}, 0<q \leq \infty, 0<p \leq u<\infty$, with $s>\frac{d}{p}$. Then

$$
e_{k}\left(\operatorname{id}_{\mathcal{A}}: \mathcal{A}_{u, p, q}^{s}(\Omega) \rightarrow L_{\infty}(\Omega)\right) \sim k^{-s / d}, \quad k \in \mathbb{N} .
$$

Proof. We apply Theorem 3.1 (i) with $s_{2}=0, u_{2}=p_{2}=\infty$ and elementary embeddings.

3.2. Approximation numbers. By a similar line of arguments as above we can prove the following corollary. The second part of it was obtained in [35].

Corollary 3.4.

(i) Let $s_{i} \in \mathbb{R}, 0<q_{i} \leq \infty, 0<p_{i} \leq u_{i}<\infty, i=1,2$, with

$$
s_{1}>s_{2} \quad \text { and } \quad p_{1} \geq u_{2} \text {. }
$$

Then

$$
a_{k}\left(\operatorname{id}_{\mathcal{A}}: \mathcal{A}_{u_{1}, p_{1}, q_{1}}^{s_{1}}(\Omega) \hookrightarrow \mathcal{A}_{u_{2}, p_{2}, q_{2}}^{s_{2}}(\Omega)\right) \sim k^{-\left(s_{1}-s_{2}\right) / d}, \quad k \in \mathbb{N} .
$$

(ii) Let $u \geq 2,0<p \leq u$, and $\frac{d}{u}<s<\frac{d}{u}+1$. Then

$$
a_{k}\left(\operatorname{id}_{\mathcal{A}}: \mathcal{A}_{u, p, q}^{s}(\Omega) \rightarrow L_{\infty}(\Omega)\right) \sim k^{-s / d+1 / u}, \quad k \in \mathbb{N} .
$$

Proof. First we apply the same arguments as above in Steps 1 and 2 of the proof of Theorem 3.1 using again the embeddings 23 and the multiplicativity of approximation numbers 13]. This leads to

$$
a_{k}\left(\operatorname{id}_{B}^{\text {out }}\right) \leq c a_{k}\left(\operatorname{id}_{\mathcal{N}}\right) \leq c^{\prime} a_{k}\left(\operatorname{id}_{B}^{\mathrm{int}}\right) .
$$

Now assume $s_{1}>s_{2}, u_{2} \leq p_{1}$, then $p_{2} \leq u_{1}$ and

$$
a_{k}\left(\operatorname{id}_{B}^{\text {out }}\right) \sim a_{k}\left(\mathrm{id}_{B}^{\text {int }}\right) \sim k^{-\left(s_{1}-s_{2}\right) / d},
$$

which leads to

$$
a_{k}\left(\operatorname{id}_{\mathcal{N}}\right) \sim e_{k}\left(\operatorname{id}_{\mathcal{N}}\right) \sim k^{-\left(s_{1}-s_{2}\right) / d}
$$

if $s_{1}>s_{2}$ and $u_{2} \leq p_{1}$. 
REMARK 3.5. In [1, Sect. 6] one can find similar results for the periodic case and more general Morrey type spaces.

Acknowledgements. The second author was supported by National Science Center, Poland, Grant No. 2013/10/A/ST1/00091.

\section{References}

[1] Z. Baituyakova, W. Sickel, Strong summability of Fourier series and generalized Morrey spaces, Anal. Math. 43 (2017), 371-414.

[2] A. Caetano, About approximation numbers in function spaces, J. Approx. Theory 94 (1998), 383-395.

[3] B. Carl, I. Stephani, Entropy, Compactness and the Approximation of Operators, Cambridge Tracts in Math. 98, Cambridge Univ. Press, Cambridge, 1990.

[4] D. E. Edmunds, W. D. Evans, Spectral Theory and Differential Operators, Oxford Math. Monogr., Clarendon Press, Oxford, 1987.

[5] D. E. Edmunds, H. Triebel, Entropy numbers and approximation numbers in function spaces, Proc. London Math. Soc. (3) 58 (1989), 137-152.

[6] D. E. Edmunds, H. Triebel, Entropy numbers and approximation numbers in function spaces II, Proc. London Math. Soc. (3) 64 (1992), 153-169.

[7] D. E. Edmunds, H. Triebel, Function Spaces, Entropy Numbers, Differential Operators, Cambridge Tracts in Math. 120, Cambridge Univ. Press, Cambridge, 1996.

[8] P. Enflo, A counterexample to the approximation problem in Banach spaces, Acta Math. 130 (1973), 309-317.

[9] D. D. Haroske, S. D. Moura, L. Skrzypczak, Smoothness Morrey spaces of regular distributions, and some unboundedness properties, Nonlinear Anal. 139 (2016), 218-244.

[10] D. D. Haroske, L. Skrzypczak, Continuous embeddings of Besov-Morrey function spaces, Acta Math. Sin. (Engl. Ser.) 28 (2012), 1307-1328.

[11] D. D. Haroske, L. Skrzypczak, Embeddings of Besov-Morrey spaces on bounded domains, Studia Math. 218 (2013), 119-144.

[12] D. D. Haroske, L. Skrzypczak, On Sobolev and Franke-Jawerth embeddings of smoothness Morrey spaces, Rev. Mat. Complut. 27 (2014), 541-573.

[13] D. Haroske, H. Triebel, Entropy numbers in weighted function spaces and eigenvalue distributions of some degenerate pseudodifferential operators I, Math. Nachr. 167 (1994), 131-156.

[14] H. König, Eigenvalue Distribution of Compact Operators, Oper. Theory Adv. Appl. 16, Birkhäuser, Basel, 1986.

[15] H. Kozono, M. Yamazaki, Semilinear heat equations and the Navier-Stokes equation with distributions in new function spaces as initial data, Comm. Partial Differential Equations 19 (1994), 959-1014.

[16] A. L. Mazzucato, Besov-Morrey spaces: function space theory and applications to non-linear PDE, Trans. Amer. Math. Soc. 355 (2003), 1297-1364.

[17] C. B. Morrey, On the solutions of quasi-linear elliptic partial differential equations, Trans. Amer. Math. Soc. 43 (1938), 126-166.

[18] J. Peetre, On the theory of $\mathcal{L}_{p, \lambda}$ spaces, J. Functional Analysis 4 (1969), 71-87.

[19] A. Pietsch, Operator Ideals, North-Holland, Amsterdam, 1980.

[20] A. Pietsch, Eigenvalues and s-Numbers, Math. Anwendungen Phys. Tech., Geest \& Portig, Leipzig, 1987. 
[21] M. Rosenthal, Local means, wavelet bases, and wavelet isomorphisms in Besov-Morrey and Triebel-Lizorkin-Morrey spaces, Math. Nachr. 286 (2013), 59-87.

[22] Y. Sawano, Wavelet characterizations of Besov-Morrey and Triebel-Lizorkin-Morrey spaces, Funct. Approx. Comment. Math. 38 (2008), 93-107.

[23] Y. Sawano, A note on Besov-Morrey spaces and Triebel-Lizorkin-Morrey spaces, Acta Math. Sin. (Engl. Ser.) 25 (2009), 1223-1242.

[24] Y. Sawano, Besov-Morrey spaces and Triebel-Lizorkin-Morrey spaces on domains, Math. Nachr. 283 (2010), 1456-1487.

[25] Y. Sawano, H. Tanaka, Decompositions of Besov-Morrey spaces and Triebel-LizorkinMorrey spaces, Math. Z. 257 (2007), 871-905.

[26] Y. Sawano, H. Tanaka, Besov-Morrey spaces and Triebel-Lizorkin-Morrey spaces for nondoubling measures, Math. Nachr. 282 (2009), 1788-1810.

[27] W. Sickel, Smoothness spaces related to Morrey spaces - a survey I, Eurasian Math. J. 3 (2012), no. 3, 110-149.

[28] W. Sickel, Smoothness spaces related to Morrey spaces - a survey II, Eurasian Math. J. 4 (2013), no. 1, 82-124.

[29] L. Tang, J. Xu, Some properties of Morrey type Besov-Triebel spaces, Math. Nachr. 278 (2005), 904-917.

[30] H. Triebel, Theory of Function Spaces, Monogr. Math. 78, Birkhäuser, Basel, 1983.

[31] H. Triebel, Theory of Function Spaces II, Monogr. Math. 84, Birkhäuser, Basel, 1992.

[32] H. Triebel, Theory of Function Spaces III, Monogr. Math. 100, Birkhäuser, Basel, 2006.

[33] H. Triebel, Local Function Spaces, Heat and Navier-Stokes Equations, EMS Tracts Math. 20, Eur. Math. Soc., Zürich, 2013.

[34] H. Triebel, Hybrid Function Spaces, Heat and Navier-Stokes Equations, EMS Tracts Math. 24, Eur. Math. Soc., Zürich, 2015.

[35] W. Yuan, D. D. Haroske, S. D. Moura, L. Skrzypczak, D. Yang, Limiting embeddings in smoothness Morrey spaces, continuity envelopes and applications, J. Approx. Theory 192 (2015), 306-335.

[36] W. Yuan, D. D. Haroske, L. Skrzypczak, D. Yang, Embedding properties of Besov-type spaces, Appl. Anal. 94 (2015), 318-340.

[37] W. Yuan, W. Sickel, D. Yang, Morrey and Campanato Meet Besov, Lizorkin and Triebel, Lecture Notes in Math. 2005, Springer, Berlin, 2010. 
